Article

\title{
Optimal Measurement Speed and Its Determination Method in the Transmission Precision Evaluation of Precision Reducers
}

\author{
Hang Xu, Zhaoyao Shi, Bo Yu * and Hui Wang \\ Beijing Engineering Research Center of Precision Measurement Technology and Instruments, \\ College of Mechanical Engineering and Applied Electronics Technology, Beijing University of Technology, \\ Beijing 100124, China; xuhangzzti@126.com (H.X.); shizhaoyao@bjut.edu.cn (Z.S.); \\ wanghui_binghai@126.com (H.W.) \\ * Correspondence: yubo@bjut.edu.cn; Tel.: +86-158-1049-5293
}

Received: 22 April 2019; Accepted: 22 May 2019; Published: 26 May 2019

check for updates

Featured Application: The method that is proposed in this paper provides a scientific basis for the speed determination in the transmission error measurement. The optimal measurement speed and its determination method are suitable for the transmission error measurement of various precision reducers.

\begin{abstract}
Transmission error is the key index for characterizing the transmission precision of precision reducers, and its accurate measurement is significant for the precision evaluation of precision reducers. Transmission error is generally measured under the conditions of zero-load and low speed. However, low speed is a general concept and there is no general standard of measurement speed or solid scientific basis. Therefore, it is difficult to obtain consistent transmission precision evaluation results for the same precision reducer. The concept of optimal measurement speed in the transmission precision evaluation of precision reducers was put forward in order to reduce the influence of measurement speed. The determination method of optimal measurement speed was proposed and the calculation model of the optimal measurement speed was established, according to the Stribeck friction model of precision reducers. Taking a certain type of RV reducer as an example, the transmission error measurement experiments were carried out under different speeds. The friction torque of the RV reducer and the peak-to-peak value of the measured transmission error were the least under the optimal measurement speed. The influence of speed on the measurement results can be effectively reduced. The determination of optimal measurement speed of transmission errors could improve the measurement precision of the transmission errors for the objective evaluation of transmission precision of precision reducers.
\end{abstract}

Keywords: precision reducer; transmission error; Stribeck curve; optimal measurement speed

\section{Introduction}

A precision reducer is the core component of an industrial robot and it directly affects the performance of a robot. RV reducers and harmonic reducers are widely used in robotics. The RV reducers have the higher rigidity and they are mostly applied in the parts of heavy loads, such as bases, arms, and shoulders, whereas the harmonic reducers are usually applied in forearms, wrists, and hands [1].

Transmission error (TE) is the key index for characterizing the transmission precision of precision reducers. It has an important influence on the positioning precision of robots and it is also the main cause for vibration. The transmission error is listed as the precision index of precision reducers in GB/T 
35089/2018 [2]. It is necessary to measure and evaluate the transmission errors of precision reducers before leaving the factory. The measurement precision of transmission errors has a significant influence on the precision evaluation of the precision reducers.

The transmission error of precision reducers has been extensively explored from the perspective of measurement and control. Gandhi et al. decomposed the transmission error of a harmonic reducer into the transmission error $\widetilde{\theta}_{p}$ that is related to manufacturing and installation errors and the transmission error $\widetilde{\theta}_{s}$ related to speed, load, and stiffness. Gandhi proposed to measure the transmission error component $\widetilde{\theta}_{p}$ under the conditions of zero-load and low speed and set the measurement speed as $10 \mathrm{r} / \mathrm{min}[3,4]$. Nye analyzed the internal and external factors that affect the transmission error of a harmonic reducer, divided the transmission error into the well-behaved transmission error and the poorly-behaved transmission error, and carried out the experimental study [5]. Lv decomposed the transmission error of a RV reducer into static error and dynamic error. Taking RV-20E as an example, the static error was obtained at $30 \mathrm{r} / \mathrm{min}$ [6]. The measurement speeds in the above studies were different, although they all divided the transmission error of a precision reducer into two components. Wang measured the transmission error of a new type of involute planetary gear transmission with double cranks and variable tooth thickness at $300 \mathrm{r} / \mathrm{min}$ [7]. Ran measured the transmission error of a $\mathrm{RV}$ reducer and compared the result with the simulation transmission error under the actual machining error [8]. Iwasaki decomposed the transmission error of a harmonic reducer into the synchronous error $\theta_{S y n c}$ and nonlinear elastic error $\theta_{H y s}$, established the mathematical models of each transmission error component, and explored the control strategy [9].

In general, the transmission error is generally measured under the conditions of zero-load and low speed and the peak-to-peak value is treated as the evaluation basis of the transmission error of precision reduces in order to realize the transmission precision evaluation of the precision reducers. However, low speed is a general concept, and there is no uniform standard, and it thus results in the difference in the transmission precision evaluation results of the same precision reducer.

From the perspective of transmission precision evaluation of precision reducers, the concept of optimal measurement speed was put forward in this paper in order to reduce the influence of the measurement speed on the dynamic measurement of transmission error. Subsequently, the calculation model of the optimal measurement speed of transmission error was established and the implementation steps were given based on the Stribeck friction model of precision reducers. Finally, taking a certain type of RV reducer as an example, with the RV reducer comprehensive performance tester that was developed by our research group, the transmission error measurement experiment was carried out to verify the proposed method.

\section{Optimal Measurement Speed in the Evaluation of Transmission Precision}

\subsection{Influencing Factors of the Transmission Error}

The transmission error of precision reducers is defined as the deviation between the actual output angle and the expected output angle of the output shaft and can be expressed as Equation (1) [2,3]. Figure 1 shows the transmission error curve and the peak-to-peak value is treated as the evaluation basis of the transmission precision of precision reduces.

$$
T E=\theta_{\text {out }}-\frac{\theta_{\text {in }}}{R}
$$

where TE is the transmission error; $\theta_{\text {out }}$ is the actual angle of the output; $\theta_{\text {in }}$ is the angle of the input; and, $R$ is the speed ratio of the precision reducer. 


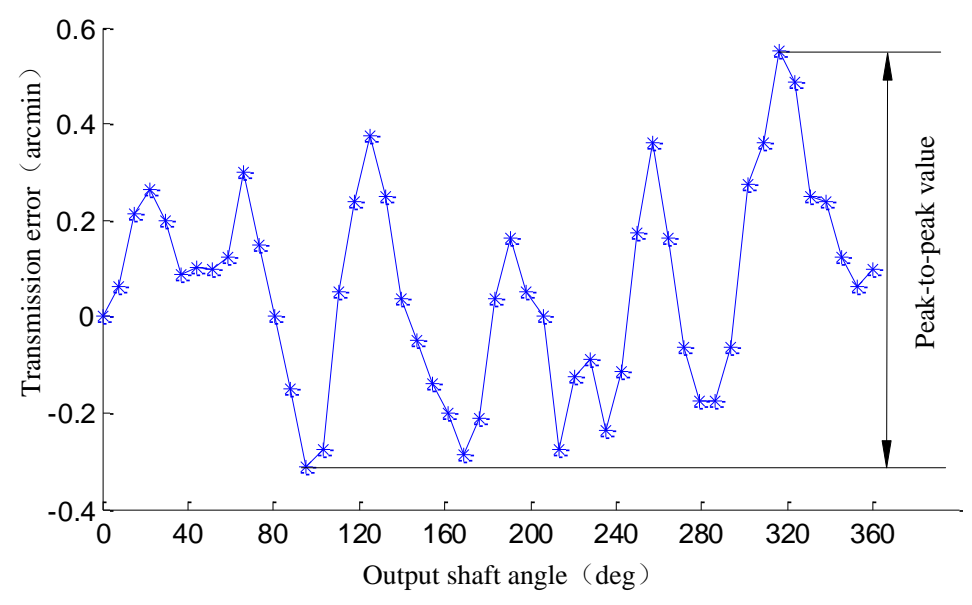

Figure 1. Transmission error curve.

The influencing factors in the dynamic measurement of a transmission error can be divided into internal and external factors. The internal factors mainly include design tolerance, manufacturing tolerance, and backlash of assembly link. The influences of internal factors are inevitable in the stage of manufacturing and assembly, and improving the manufacturing and assembly precision can decrease them. External factors, including speed, load torque, and temperature, are closely related to measurement conditions. The transmission error measurement is usually done within a short time, so the influence of temperature can be ignored.

\subsection{Optimal Measurement Speed}

Transmission error is the main basis for evaluating the transmission precision of a precision reducer. The key to its measurement is to avoid or minimize the influence of external factors as much as possible. At present, the transmission error measurement is mainly realized under the conditions of zero-load and low speed. The purpose of zero-load setting is to avoid the influence of elastic deformation that is caused by load torque and the purpose of low speed is to reduce the influence of friction torque of a precision reducer. The concept of optimal measurement speed is put forward in this paper from the perspective of reducing the influence of friction torque of precision reducers.

The friction torque of precision reducers decreases with the increase in the speed when the speed is low. The friction torque increases with the increase in the speed when the speed is greater than a certain value, showing a typical Stribeck effect (Figure 2) [10-13]. It can be seen that the forward and reverse Stribeck curves of precision reducers have a wave trough point $A^{+}$and a wave peak point $A^{-}$, respectively. Taking the forward curve as an example, when the speed $\omega \leq \omega_{A}^{+}$, the friction torque of precision reducers decreases with the increase in speed. When the speed $\omega \geq \omega_{A}^{+}$, the friction torque of precision reducers increases with the increase in the speed. When the speed $\omega=\omega_{A}^{+}$, the precision reducer is subjected to the smallest friction torque. Therefore, the friction torque is the smallest when the speed $\omega=\omega_{A}^{+}$, and this speed is the optimal speed for the forward transmission error measurement. Similarly, $\omega=\omega_{A}^{-}$is the optimal speed for the reverse transmission error measurement.

Therefore, the influences of external factors can be effectively reduced under the conditions of zero-load and optimal measurement speed and the objective evaluation of transmission precision can be realized in the dynamic measurement of the transmission error of a precision reducer. The transmission error can be expressed as:

$$
T E= \begin{cases}F\left(\omega_{A}^{+}, T_{0}\right) & \omega>0 \\ F\left(\omega_{A}^{-}, T_{0}\right) & \omega<0\end{cases}
$$

where $F\left(\omega_{A}^{+}, T_{0}\right)$ is the forward transmission error curve under the conditions of zero-load and optimal measurement speed $\omega_{A}^{+}$and $F\left(\omega_{A^{-}}^{-} T_{0}\right)$ is the reverse transmission error curve under the conditions of zero-load and optimal measurement speed $\omega_{A}^{-}$. 


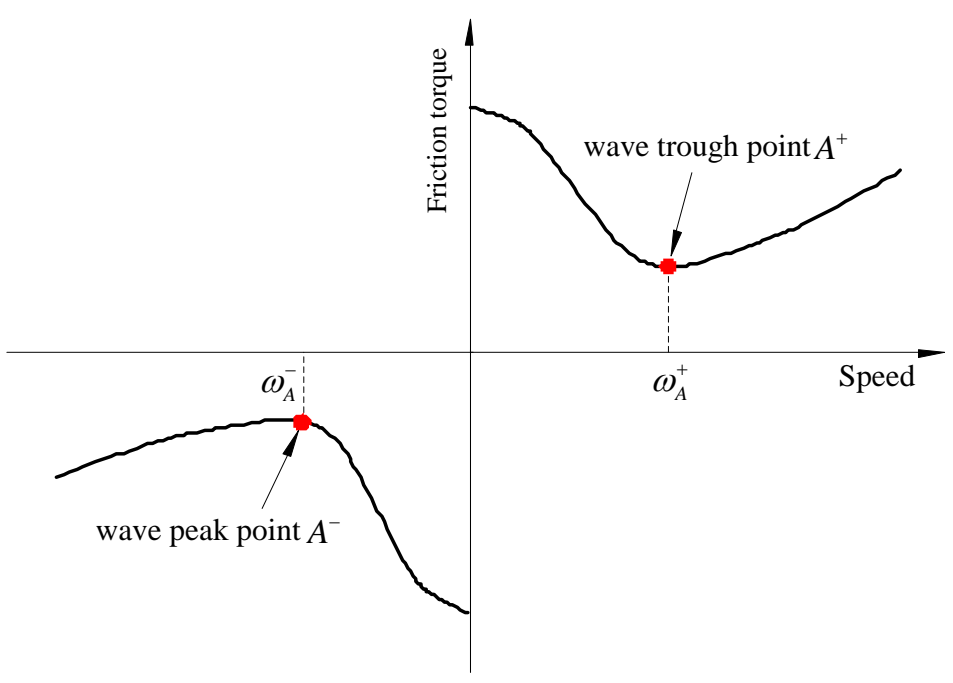

Figure 2. Optimal measurement speed.

\section{Determination Method of Optimal Measurement Speed}

\subsection{Friction Model of Precision Reducers}

The friction characteristics of precision reducers can be divided into the following four stages according to the Stribeck friction theory: (I) static friction stage, (II) boundary lubrication stage, (III) partial fluid lubrication stage, and (IV) full fluid lubrication stage, as shown in Figure 3. Firstly, the precision reducer is ready to start and it is in the static friction stage when the speed of the precision reducer is zero. The friction is the static friction independent of the speed and it is mainly caused by the elastic deformation of a precision reducer. Secondly, with the increase in external force, there is a low relative speed between the contact surfaces and the lubricating oil film cannot be established between the surfaces. The friction is mainly determined by the impurity characteristics of the boundary layer and the shearing force between solids causes it. At this time, the precision reducer is in the boundary lubrication stage. Thirdly, with the further increase in the speed, the liquid film formed between the contact surfaces is thicker and thicker, but the contact surfaces are not completely separated by the lubricant and there is still a region of solid contact, which indicates the partial fluid lubrication stage. Fourthly, the liquid film between the objects is completely formed when the speed continues to increase, and the region of solid contact does not exist. However, with the increase in the relative speed, the viscous friction, which is proportional to the speed, is the dominant friction. At this time, the friction mainly depends on the speed and the viscosity coefficient of the lubricant and it enters the stage of full fluid lubrication [14,15].

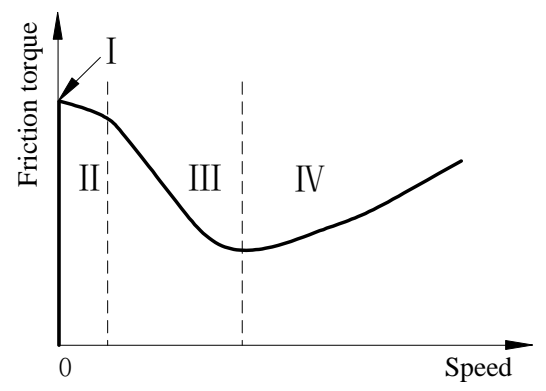

Figure 3. Four friction stages of precision reducers: (I) static friction stage, (II) boundary lubrication stage, (III) partial fluid lubrication stage, and (IV) full fluid lubrication stage.

In this study, the Tustin empirical model is used to describe the Stribeck friction effect of precision reducers $[16,17]$. The clockwise rotation of precision reducers is defined as the forward direction 
and the counterclockwise rotation is defined as the reverse direction. The friction model of precision reducers is established, as follows:

$$
\begin{cases}T_{f}^{+}(\omega)=T_{c}^{+}+\left(T_{s}^{+}-T_{c}^{+}\right) \cdot e^{-\left(\omega / \omega_{s}^{+}\right)}+B^{+} \cdot \omega & \omega>0 \\ T_{f}^{-}(\omega)=-T_{c}^{-}-\left(T_{s}^{-}-T_{c}^{-}\right) \cdot e^{-\left(\omega / \omega_{s}^{-}\right)}+B^{-} \cdot \omega & \omega<0\end{cases}
$$

where $T_{f}^{+}(\omega)$ and $T_{f}^{-}(\omega)$, respectively, indicate the forward and reverse friction torques; $T_{c}^{+}$and $T_{c}^{-}$, respectively, denote forward and reverse Coulomb friction torques; $T_{s}^{+}$and $T_{s}^{-}$, respectively, denote forward and reverse static friction torques; $W_{s}^{+}$and $W_{s}^{-}$, respectively, denotes forward and reverse Stribeck speeds; and, $B^{+}$and $B^{-}$, respectively, denote the forward and reverse viscous friction coefficients.

\subsection{Calculation Model of the Optimal Measurement Speed}

It can be seen from Equation (3) that the forward and reverse friction functions are continuous and can be respectively derived, so the coordinates of the points $A^{+}$and $A^{-}$can be solved with the first order differential equation of Equation (3). Equation (4) shows the first order differential equation of Equation (3). If Equation (4) is equal to zero, then the optimal measurement forward and reverse speed can be obtained with Equation (5).

$$
\begin{gathered}
T_{f}^{\prime}(\omega)=\left\{\begin{array}{cc}
-\frac{1}{\omega_{s}^{+}} \cdot\left(T_{s}^{+}-T_{c}^{+}\right) e^{-\left(\omega / \omega_{s}^{+}\right)}+B^{+} & \omega>0 \\
\frac{1}{\omega_{s}^{-}} \cdot\left(T_{s}^{-}-T_{c}^{-}\right) e^{-\left(\omega / \omega_{s}^{-}\right)}+B^{-} & \omega<0
\end{array}\right. \\
\left\{\begin{array}{l}
\omega_{A}^{+}=-\omega_{s}^{+} \ln \frac{B^{+} \cdot \omega_{s}^{+}}{T_{s}^{+}-T_{+}^{+}} \\
\omega_{A}^{-}=-\omega_{s}^{-} \ln \frac{-B_{s}^{-} \cdot \omega_{s}^{-}}{T_{s}^{-}-T_{c}^{-}}
\end{array}\right.
\end{gathered}
$$

\subsection{Steps for Determining the Optimal Measurement Speed}

The steps for determining the optimal measurement speed of transmission errors in this paper are provided, as follows:

(1) First of all, the friction torque of the precision reducer is measured under different speeds. The selected measurement speed should range from the low speed to high speed, so that it can fully reflect the Stribeck friction effect of the precision reducer.

(2) According to the measured data of friction torque and speed of a precision reducer, the Stribeck curve is drawn and the parameters of the Stribeck model are obtained by curve fitting.

(3) The optimal measurement speed of transmission errors is calculated by substituting the parameter value of the Sribbeck model into Equation (5).

(4) The transmission error of a precision reducer is measured and evaluated under the conditions of zero-load and optimal measurement speed.

\section{Experimental Study}

Taking a RV reducer as an example, based on the RV reducer comprehensive performance tester that was developed by our research group, the transmission error measurement experiment was carried out. The rated torque of the measured RV reducer is $784 \mathrm{~N} \mathrm{~m}$ and the speed ratio is 121 . The number of cycloidal gear teeth is 39 and the number of pin gears is 40 .

Our research group developed a RV reducer comprehensive performance tester (Figure 4) [14,18]. The RV reducer was installed on the precision support and the input shaft was driven by a servo motor in the transmission error measurement. The measuring and controlling software in the speed mode controlled the measurement speed to ensure the speed stability in the measurement. The dynamic measurement of transmission error can be realized by synchronously collecting the rotation angle 
signal with high precision circular grating installed at the input and output ends. In the transmission error measurement process, the torque sensor installed at the input end measured the friction torque.

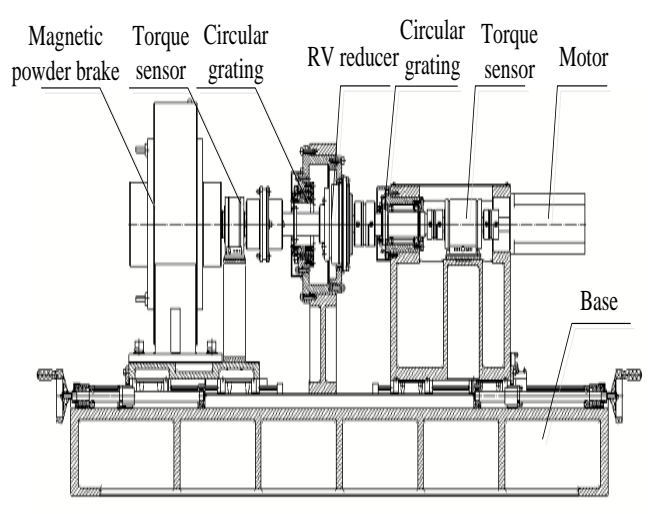

(a)

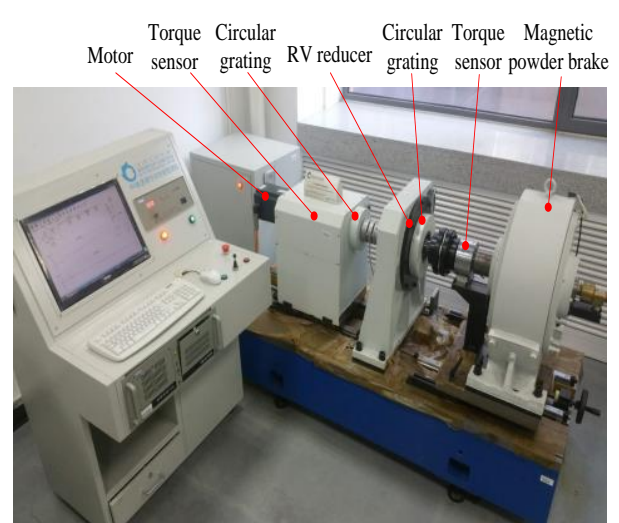

(b)

Figure 4. RV reducer comprehensive performance tester. (a) Structure diagram; and, (b) Physical view.

\subsection{Stribeck Curve Measurement and Parameter Identification}

This section provides a concise and precise description of the experimental results, interpretation, and conclusions. In order to accurately obtain the Stribeck friction curve of the precision reducer, the friction torque measurement experiment was carried out under different speed conditions, from low speed to high speed. When the drive motor works in the speed mode, the speed fluctuation is very small and it can be approximately ignored. The friction torque of the precision reducer is equal to the drive torque of the motor at the input end, which is acquired by the torque sensor at the input end in real time. Figure 5 shows the variation of the friction torque with the meshing position under a speed of $2.0933 \mathrm{rad} / \mathrm{s}$. In order to obtain the one-to-one corresponding relationship between the friction torque and speed, the mean value filtering method is used to deal with the measured data of friction torque under a steady speed [19]. In other words, the mean value of friction torque is used as the corresponding friction torque under steady speed. This method can also filter out the noise interference at the same time.

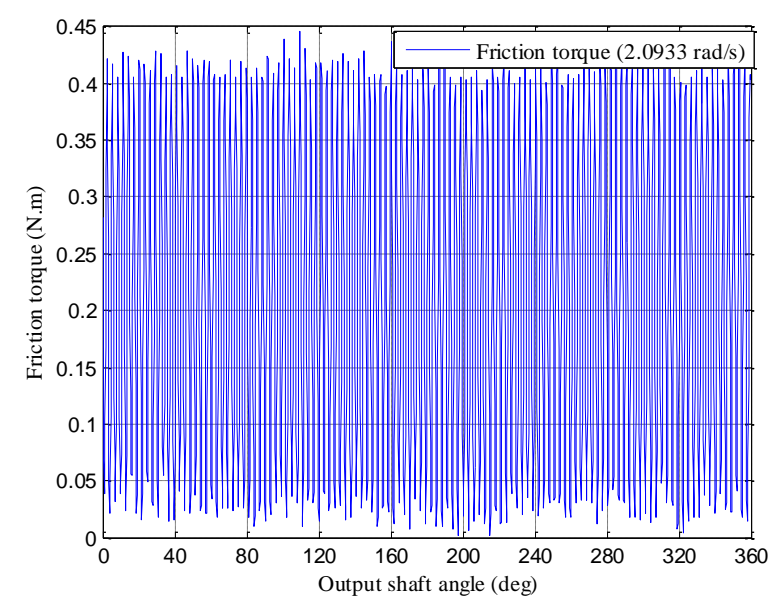

Figure 5. Friction torque of a RV reducer $(2.0933 \mathrm{rad} / \mathrm{s})$.

The friction torque-speed data of the RV reducer were obtained by changing the measurement speed (Table 1). The Stribeck curve of the precision reducer was fitted with the least square method (Figure 6). Table 2 shows the parameters of the Stribeck model that were obtained by least square fitting. The RSS (residual sum of squares) is $5.10453 \times 10^{-4}$ and the Adj. R-Square (adjusted coefficient 
of determination) is 0.97998 . The two parameters indicated that the forward fitting could achieve the ideal fitting effect.

Table 1. Measurement results of the friction torque of a RV reducer.

\begin{tabular}{cccccc}
\hline Speed (rad/s) & $\begin{array}{c}\text { Friction } \\
\text { Torque (N·m) }\end{array}$ & Speed (rad/s) & $\begin{array}{c}\text { Friction } \\
\text { Torque (N·m) }\end{array}$ & Speed (rad/s) & $\begin{array}{c}\text { Friction } \\
\text { Torque (N·m) }\end{array}$ \\
\hline 0.4187 & 0.3474 & 3.1400 & 0.2021 & 10.4667 & 0.2286 \\
0.6280 & 0.2781 & 4.1867 & 0.2008 & 12.5600 & 0.2408 \\
0.8373 & 0.2473 & 5.2333 & 0.2057 & 15.700 & 0.2580 \\
1.0467 & 0.2350 & 6.2800 & 0.2084 & 17.4444 & 0.2686 \\
1.5700 & 0.2084 & 7.3267 & 0.2137 & 20.9333 & 0.2889 \\
2.0933 & 0.2088 & 8.7222 & 0.2199 & 26.1667 & 0.3187 \\
\hline
\end{tabular}

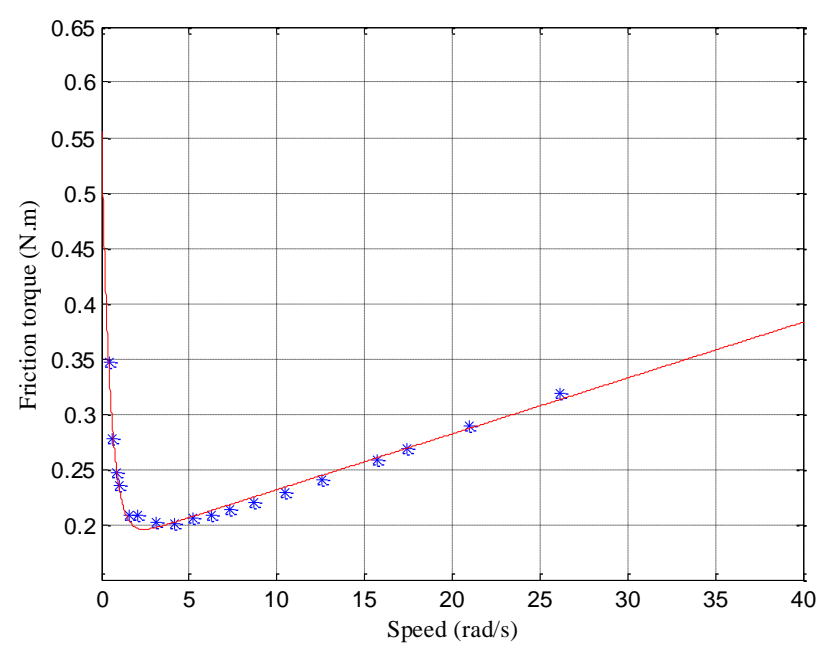

Figure 6. Fitted Stribeck curve of a RV reducer.

Table 2. Fitted parameters of the Stribeck model of a RV reducer.

\begin{tabular}{ccccccc}
\hline Parameters & $\boldsymbol{T}_{c}^{+}$ & $\boldsymbol{T}_{s}^{+}$ & $\omega_{s}^{+}$ & $\boldsymbol{B}^{+}$ & RSS & Adj. R-Square \\
\hline Values & 0.18081 & 0.55604 & 0.48697 & 0.00505 & $5.10453 \times 10^{-4}$ & 0.97998 \\
\hline
\end{tabular}

\subsection{Transmission Error Measurement}

After substituting the parameters of the fitted Stribeck curve into Equation (5), the forward optimal measurement speed can be calculated as $\omega_{A}^{+}=2.4483 \mathrm{rad} / \mathrm{s}$, according to the determination method of the optimal measurement speed of the transmission error of a precision reducer proposed in this paper. Taking the forward direction as an example, the transmission error measurement experiment was carried out. First, the transmission error of the precision reducer was measured under the conditions of zero-load and optimal measurement speed. The peak-to-peak value was 0.5058 arcmin (Figure 7). The transmission error of the precision reducer was measured when the measurement speed was changed and the other conditions remained unchanged. Table 3 shows the peak-to-peak values of transmission error of precision reducer measured at different speeds. Figure 8 shows the variation of the peak-to-peak value of the transmission error with measurement speed. Under the optimal measurement speed, the peak-to-peak value of transmission error was the smallest, because the friction torque of the precision reducer, as well as its influence on transmission errors, was the smallest under the optimal measurement speed. Therefore, the influences of external factors were reduced to the largest degree under the conditions of optimal measurement speed and zero-load. In this way, the objective measurement and evaluation results of transmission precision were realized. 


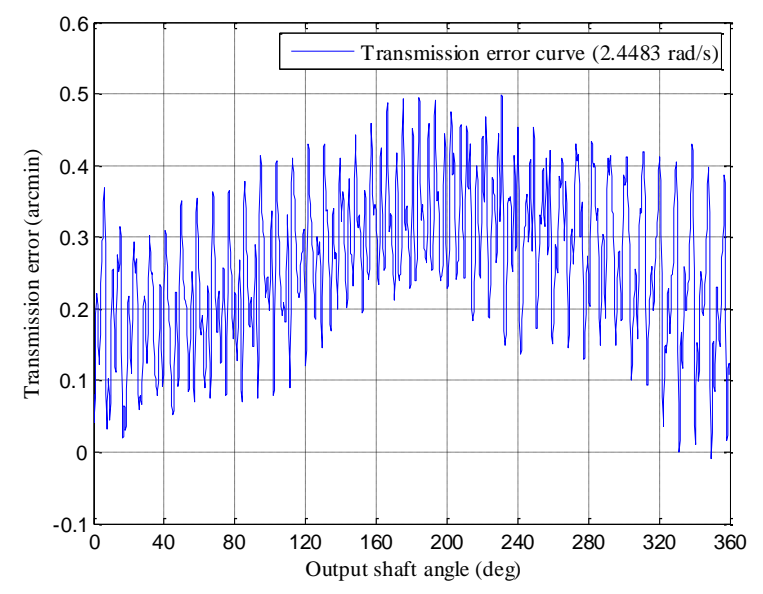

Figure 7. Transmission error curve (2.4483 rad/s).

Table 3. Peak-to-peak values of transmission errors under different speeds.

\begin{tabular}{cccccc}
\hline $\begin{array}{c}\text { Speed } \\
(\mathrm{rad} / \mathrm{s})\end{array}$ & $\begin{array}{c}\text { Peak-to-Peak } \\
\text { Value (Arcmin) }\end{array}$ & $\begin{array}{c}\text { Speed } \\
(\mathrm{rad} / \mathbf{s})\end{array}$ & $\begin{array}{c}\text { Peak-to-Peak } \\
\text { Value (Arcmin) }\end{array}$ & $\begin{array}{c}\text { Speed } \\
\text { (rad/s) }\end{array}$ & $\begin{array}{c}\text { Peak-to-Peak } \\
\text { Value (Arcmin) }\end{array}$ \\
\hline 0.4187 & 0.6119 & 2.4483 & 0.5058 & 10.4667 & 0.5318 \\
0.8373 & 0.6068 & 3.1400 & 0.5141 & 12.5600 & 0.5391 \\
1.0467 & 0.5631 & 4.1867 & 0.5163 & 17.4444 & 0.5409 \\
1.5700 & 0.5541 & 5.2333 & 0.5165 & 20.9333 & 0.5468 \\
2.0933 & 0.5283 & 7.3267 & 0.5246 & 26.1667 & 0.6076 \\
\hline
\end{tabular}

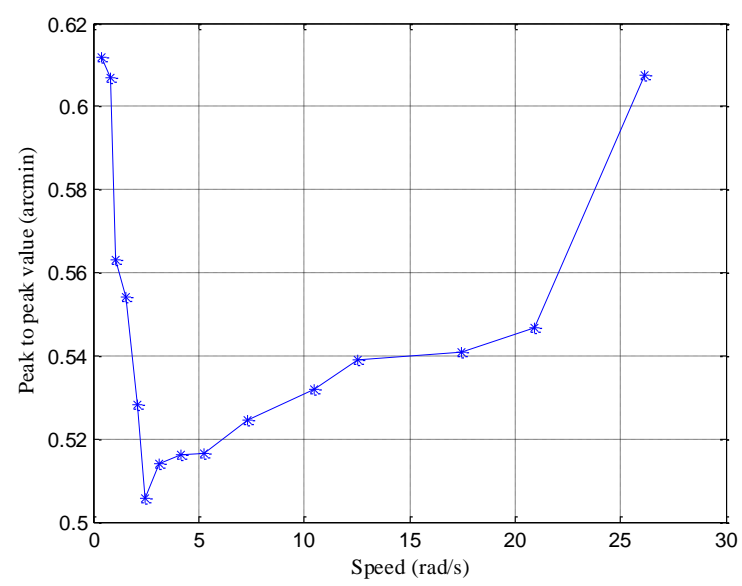

Figure 8. Variations of the peak-to-peak value of transmission errors with speed.

\subsection{Spectrum Analysis}

The spectrum analysis of transmission error under the optimal measurement speed was carried out based on the spatial spectrum analysis method [20]. In order to more accurately obtain the error source, the low-pass filtering of the original transmission error curve was carried out. The spectrum of the filtered transmission error is shown in Figure 9, where $f$ and $f_{0}$, respectively, represent the frequency of transmission error and the frequency of output shaft; $f / f_{0}$ represents the number of variation times of transmission error within one rotation of the output shaft [21]. Table 4 shows the harmonic components. The spectral component contains a constant term. The constant term is mainly caused by the inherent clearance of the RV reducer. In the transmission error measurement of the RV reducer, the pin gear shell was fixed and the planetary frame was the output end. Therefore, in the range of one rotation of the output shaft, the planetary frame rotated in a circle and the harmonic component 1 was mainly caused by the machining error of the planetary frame. The number of 
cycloidal gear teeth of the measured RV reducer was 39 and the harmonic components 39 and 78 were mainly caused by the machining error of the cycloidal gear. The number of pin gear of the measured RV reducer was 40, and the harmonic components 40, 80, 160, and 200 were mainly caused by the machining error of a pin gear. The input shaft rotated 121 cycles in the range of one rotation of the output shaft, and the harmonic component 121 was mainly caused by the machining error of input shaft. The harmonic components indicated that the machining and installation errors of planetary frame, cycloid gear, pin gear, and input shaft of the RV reducer had great influences on transmission errors and it should be considered in the design and manufacturing process.

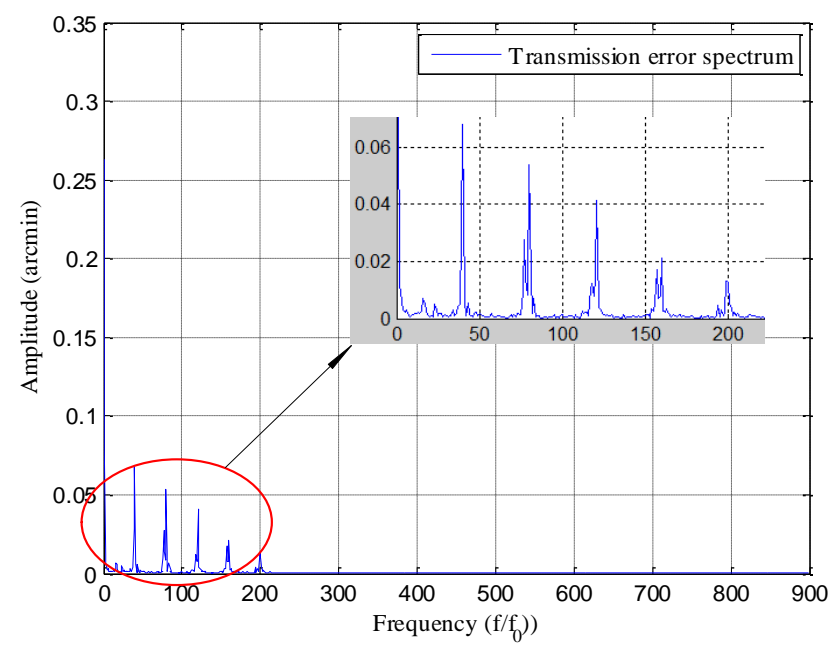

Figure 9. Transmission error spectrum.

Table 4. Harmonic components of the transmission error.

\begin{tabular}{cccccc}
\hline $\begin{array}{c}\text { Harmonic } \\
\text { Components }\end{array}$ & Amplitudes & $\begin{array}{c}\text { Harmonic } \\
\text { Components }\end{array}$ & Amplitudes & $\begin{array}{c}\text { Harmonic } \\
\text { Components }\end{array}$ & Amplitudes \\
\hline 0 & 0.2628 & 40 & 0.0678 & 121 & 0.0412 \\
1 & 0.0790 & 78 & 0.0140 & 160 & 0.0214 \\
39 & 0.0283 & 80 & 0.0540 & 200 & 0.0128 \\
\hline
\end{tabular}

\section{Discussion}

The friction torque of the precision reducers decreases with the increase in the speed when the speed is low. The friction torque increases with the increase in the speed when the speed is greater than a certain value, displaying the typical Stribeck friction effect.

The friction torque of the precision reducer and the peak-to-peak value of the measured transmission error are the least under the optimal measurement speed. The influence of speed on the measurement results can be effectively reduced. The objective measurement and evaluation of transmission precision of precision reducer can be realized under the conditions of zero-load and optimal measuring speed.

The speed only affects the amplitudes of harmonic components of the transmission error other than the harmonic component in the transmission error measurement. The speed is the external factor of transmission error. The harmonic component of transmission error is mainly determined by the internal structure of the precision reducer.

\section{Conclusions}

In this paper, the concept of optimal measurement speed in the transmission precision evaluation of precision reducers was put forward. The calculation model of optimal measurement speed was 
established based on the Stribeck friction model of precision reducers. The following conclusions can be obtained through measurement experiments while taking the RV reducer as an example.

(1) The influence of speed on the transmission error measurement of the precision reducer is objective and the scientific and reasonable measurement speed can reduce the influence of the friction torque to the largest degree.

(2) The transmission error measurement of the precision reducer under the conditions of optimal measurement speed and zero-load can improve the transmission error measurement precision and realize the objective transmission precision evaluation of the precision reducer.

(3) The method that is proposed in this paper provides a scientific basis for the speed determination in the transmission error measurement.

(4) The optimal measurement speed and its determination method that are presented in this paper are applicable to measure the transmission error of various precision reducers.

Author Contributions: Conceptualization, Z.S. and H.X.; methodology, Z.S. and H.X.; validation, B.Y. and H.X.; formal analysis, H.X.; data curation, H.W.; writing—original draft preparation, H.X.; writing—review and editing, H.X. and B.Y.; funding acquisition, Z.S. and B.Y.

Funding: Please add: This research was supported by the National Natural Science Foundation of China (No. 51805011, 51635001), and the National High Technology Research and Development Program 863 of China (No. 2015AA043002).

Conflicts of Interest: The authors declare no conflict of interest.

\section{References}

1. Shi, Z.Y.; Xu, H.; Han, F.X.; Lin, J.C.; Wang, H. Current status and trends in precision reducer lost motion measurement. Opt. Precis. Eng. 2018, 26, 11-20.

2. GB/T 35089-2018. Precision Gear Transmission for Robot-Test Method; Standardization Administration of the People's Republic of China: Beijing, China, 2018.

3. Ghorbel, F.H.; Gandhi, P.S.; Alpeter, F. On the kinematic error in harmonic drive gears. ASME. J. Mech. Des. 1998, 123, 90-97. [CrossRef]

4. Gandhi, P.S. Modeling and Control of Nonlinear Transmission Attributes in Harmonic Drive Systems. Ph.D. Thesis, Rice University, Houston, TX, USA, 2001.

5. Nye, T.; Kraml, R. Harmonic drive gear error: Characterization and compensation for precision pointing and tracking. In Proceedings of the 25th Aerospace Mechanics Symposium, Washington, DC, USA, January 1991; pp. 237-252.

6. Lv, M.S. Simulation and Experimental Study of Transmission Characteristic of RV Reducer. Master's Thesis, Harbin Institute of Technology, Harbin, China, 2016.

7. Wang, G.J.; Jiang, H.J. Decelopment and test of new double-crank conical involute planetary gears. J. Mach. Des. 2011, 28, 62-65.

8. Ran, Y. Analysis of Transmission Precision for RV Reducer. Master's Thesis, Chongqing University, Chongqing, China, 2015.

9. Iwasaki, M.; Yamamoto, M.; Hiral, H.; Okitsu, Y.; Sasaki, K.; Yajima, T. Modeling and compensation for angular transmission error of harmonic drive gearings in high precision positioning. In Proceedings of the IEEE/ASME International Conference on Advanced Intelligent Mechatronics, Singapore, 14-17 July 2009; pp. 662-667.

10. Stribeck, R. Die wesentlichen eigenschaften der gleit und rollenlager: The key qualities of sliding and roller bearings. Z. Ver. Dtsch. Ing. 1902, 46, 1342-1348, 1432-1437.

11. Zhang, X.G. Researches of Experimental Modeling and System Dynamics on Frictions Concerning Extended Stribeck Effect. Ph.D. Thesis, Shanghai Jiao Tong University, Shanghai, China, 2009.

12. Yin, Z.X. Studies on Transmission Precision and Dynamic Characteristics of Harmonic Reducer. Master's Thesis, China University of Geosciences, Beijing, China, 2015.

13. Hei, M. Research on the Modeling and Control of Precision Harmonic Drive System. Ph.D. Thesis, National University of Defense Technology, Changsha, China, 2015. 
14. Wang, X.L. Development of RV Reducer Performance Tester. Master's Thesis, Beijing University of Technology, Beijing, China, 2015.

15. Marton, L.; Lantos, B. Identification and model-based compensation of Striebeck friction. Acta Polytech. Hung. 2006, 3, 45-58.

16. Tustin, A. The effects of backlash and of speed dependent friction on the stability of closed-cycle contral systems. J. Inst. Electr. Eng. 1947, 94, 143-151.

17. Marton, L.; Lantos, B. Modeling, identification, and compensation of Stick-Slip friction. IEEE Trans. Ind. Electron. 2007, 54, 511-521. [CrossRef]

18. Shi, Z.Y.; Xu, H.; Lin, J.C.; Yu, B.; Wang, H. Research on measurement and evaluation of precision reducer lost motion. Chin. J. Sci. Instrum. 2018, 39, 56-63.

19. Zhao, Z.Q. Software Design on Nonlinear Parameters Measurement System of Steering Gear Reducer. Master's Thesis, Harbin Institute of Technology, Harbin, China, 2015.

20. Yan, S.D.; Liu, Z.M. Transmission precision of experimental tests and analysis of a new type of reducer. Mod. Manuf. Eng. 2015, 11, 124-127.

21. Han, L.S.; Shen, Y.W.; Dong, H.J.; Wang, G.F.; Liu, J.Y.; Qi, H.J. Theoretical research on dynamic transmission precision for 2K-V-type drive. Chin. J. Mech. Eng. 2007, 81-86. [CrossRef]

(C) 2019 by the authors. Licensee MDPI, Basel, Switzerland. This article is an open access article distributed under the terms and conditions of the Creative Commons Attribution (CC BY) license (http://creativecommons.org/licenses/by/4.0/). 